\title{
Corrosion Protection of Steel by Calcareous Electrodeposition in Seawater (Part 4)
}

\author{
- Method of Proper Anodes Arrangement- \\ Isamu Kashiki *, Akira Suzuki * and Kenichi Akamine ** \\ * Graduate School of Fisheries Science, Hokkaido University \\ ** Material Processing \& Evaluation Group, Production Engineering Department, \\ Advanced Production Engineering Center, Ishikawajima-Harima Heavy Industries Co., Ltd.
}

\begin{abstract}
To estimate a proper anodes arrangement in the cathodic protection of a gigantic marine structure, quite a simple method was proposed, and the validity and applicability of the method was examined. The method was based on the assumption that the electric resistance between an anode and the structure mainly come from the part of seawater in cathodic protection of a gigantic marine structure. To examine the validity of this assumption, the potentials of the anode and cathode under the cathodic protection at a constant current density were measured using seawaters diluted to various salinities. The results showed that the dilution method is valid for the estimation of the anodes arrangement in the cathodic protection of gigantic marine structures.

To show how to apply the dilution method to a concrete design problem in protecting a gigantic marine structure cathodically, the electric current distributions on a structure during cathodic protection were measured in seawater diluted to $1 / 10$ with fresh water. A close relation was found between the distribution of current density and that of accompanying calcareous deposit amount. The current density data were treated statistically, and a dimensionless number was introduced to evaluate quantitatively the unevenness of the calcareous deposit distribution. It was found that the dimensionless number is very useful for designing the arrangement of electrodes for the cathodic protection of a gigantic marine structure accompanied with calcareous deposition.
\end{abstract}

Key words : steel, cathodic protection, gigantic structure, anode, arrangement, dilution, potential distribution

\section{Introduction}

The preceding paper ${ }^{1)}$ dealt with the effects of seawater dilution on the properties of calcareous deposits formed on ferrous marine structures cathodically protected. There, we concluded that, in principle, the method of cathodic protection (CP) accompanied with calcareous deposition is applicable to ferrous structures in estuary areas with the salinity more than $1 / 10$ of neat seawater.

Recently, it is often the case that ferrous structures constructed in such estuary areas are gigantic. In general, numerous anodes must be positioned for proper cathodic protection of such a gigantic marine structure; otherwise, there will arise a great unevenness in the potential distribution along the gigantic structure during $\mathrm{CP}$, and so will do in the distribution of calcareous deposit formed on the cathode surface. Because, in the method of cathodic protection $(\mathrm{CP})$, the potential and the calcareous deposit on marine structures to be protected play decisive parts ${ }^{2,3)}$, it is not desirable that there arises too much unevenness in the distribution of the potential over the structures. Hence, in designing the cathodic protection of a gigantic marine ferrous structure, it is important to estimate a necessary number of anodes and their proper arrangement to attain a uniform potential distribution over the structure. But the problem is rather difficult. First, in view of a necessary space, it is difficult to realize a simulated experiment with a

\footnotetext{
* Minato-cho 3-1-1, Hakodate, 041-8611 Japan

** Shin-nakahara-cho, Isogoku, Yokohama, 235-8501 Japan
}

gigantic structure in a laboratory. In contrast, for a field experiment in natural seawater, quite a different difficulty will arise. In this case, the flow of water varies ceaselessly the thickness of Nernst diffusion layer adjacent to a cathode $^{4), 5)}$, and a stable potential distribution can hardly be formed along a cathode. Further, this difficulty increases with increasing the geometrical complexity of the structure under consideration. Therefore, in fact, the problem of the necessary number of anodes and their arrangement has been left to the trial-and-error experiences of corrosion engineers.

The objective of this paper is to present a method of solving this problem by a simple laboratory experiment, and to supply some necessary information for the design of the cathodic protection of a gigantic marine structure.

\section{Theoretical consideration}

A schematic arrangement of the electrodes in seawater is presented in Fig. 1, where a wire-type cathode as a simulated marine structure is on the $x$-axis, and a $\mathrm{Mg}$ anode on the $y$-axis. $-x_{\mathrm{e}}$ and $x_{\mathrm{e}}$ are the points of the two ends of the wire cathode, and $x_{\mathrm{i}}$ and $y_{0}$ represent an arbitrary point on the cathode and a fixed position of the anode on the $y$-axis, respectively. Let $E_{\mathrm{Mg}}$ and $E_{\mathrm{i}}$ denote the electric potentials at $y_{0}$ of the anode and at $x_{\mathrm{i}}$ on the cathode, respectively. In the circuit in Fig. 1, the electric resistance can be considered to come from the three parts ${ }^{6}$ : the parts of cathode, anode and electrolytic solution (i.e., seawater). If the part of electrolytic solution is gigantic and the cathodic current density $\left(D_{\mathrm{k}}\right)$ is not so high, the first two parts can be 


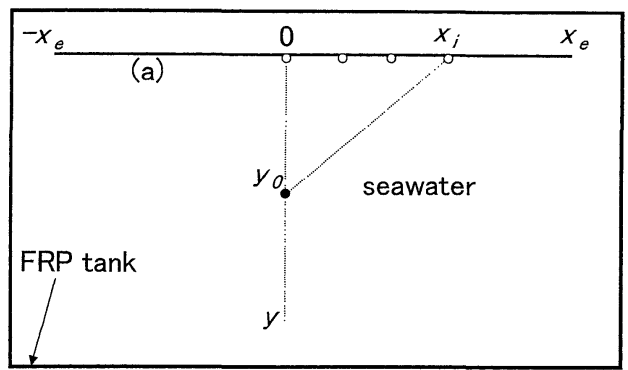

Fig. 1 Schematic diagram for arrangement of a cathode and an anode; (a): a wire-type cathode, 0 : an anode, $-x_{\mathrm{e}}$ and $x_{\mathrm{e}}$ : two end positions of the cathode, $x_{\mathrm{i}}$ : an arbitrary position on the wire-type cathode, $y_{0}$ : a fixed position of the anode, $\bigcirc$ : the points for measuring potentials on the cathode.

neglected as compared with the last one at least in the initial period of CP. Further, the anode is assumed to be so small compared with the cathode that the anode position can be approximately specified with a single point. Under these conditions ${ }^{7)}$,

$$
V_{t}=E_{\mathrm{Mg}}-E_{\mathrm{i}}+i_{\mathrm{i}} \frac{\sqrt{x_{\mathrm{i}}^{2}+y_{0}^{2}}}{k}
$$

where $V_{\mathrm{t}}$ is the voltage impressed externally by a galvanostat to maintain a constant electric current, and $k$ and $i_{\mathrm{i}}$ are the specific electric conductivity of used seawater and the current density of the wire cathode at $x=x_{\mathrm{i}}$, respectively. Rearrangement of Equation (1) gives

$$
i_{\mathrm{i}}=\frac{k\left(E_{\mathrm{i}}-V_{\mathrm{a}}\right)}{\sqrt{x_{\mathrm{i}}^{2}+y_{0}^{2}}}
$$

where the terms of $E_{\mathrm{Mg}}$ and $V_{\mathrm{t}}$ in Equation (1) which are considered to have no potential distributions are put together as

$$
V_{\mathrm{a}} \equiv E_{\mathrm{Mg}}-V_{\mathrm{t}}
$$

The value of $k$ at the salinity of $3.2 \%$ and the temperature of $20^{\circ} \mathrm{C}$ is $4.4192 \mathrm{~S} / \mathrm{m}^{8}$. If the original seawater is diluted with fresh water, the specific conductivity changes from $k$ to $d k$, where $d$ is the dilution ratio of original seawater, defined by

$$
d=C / C_{0}
$$

where $C_{0}$ and $C$ are the salinities of neat and diluted seawaters, respectively. Hence, if we take into account this dilution effect, Equation (2) is modified to

$$
i_{\mathrm{i}}=\frac{d k\left(E_{\mathrm{i}}-V_{\mathrm{a}}\right)}{\sqrt{\left(x_{\mathrm{i}}\right)^{2}+\left(y_{0}\right)^{2}}}=\frac{k\left(E_{\mathrm{i}}-V_{\mathrm{a}}\right)}{\sqrt{\left(X_{\mathrm{i}}\right)^{2}+\left(Y_{0}\right)^{2}}}
$$

where $X_{\mathrm{i}}\left(\equiv x_{\mathrm{i}} / d\right)$ and $Y_{0}\left(\equiv y_{0} / d\right)$ are, so to speak, kinds of hypothetical distances corresponding to the real distances of $x_{\mathrm{i}}$ and $y_{0}$, respectively, when some dilution is made for original seawater. Equation (5) can be interpreted in the two manners; the dilution makes the specific conductivity decrease from $k$ to $d k$, or the dilution makes the spatial positions of the electrodes hypothetically enlarge from $x_{\mathrm{i}}$ and $y_{0}$ to $X_{\mathrm{i}}\left(\equiv x_{\mathrm{i}} / d\right)$ and $Y_{0}\left(\equiv y_{0} / d\right)$. These two interpretations are equivalent mathematically, but the latter suggests that the dilution of seawater may serve as a method of estimating proper anodes arrangement in a space-saving manner. It is true that the dilution has a considerable effect on the chemical composition of electrodeposited calcareous film on a cathode, as described in the preceding paper ${ }^{1)}$. However, the CP method accompanied with calcareous deposits is applicable, in principle, so long as the dilution does not make the salinity of seawater be less than $1 / 10$ of the standard seawater value $(3.48 \%)$. Hence, hereafter, we will inspect the validity of the above scale-up Equation (5) within the dilution range of $d=0.1 \sim 1$, and how to apply the dilution method to a concrete design problem in protecting a gigantic marine structure cathodically.

\section{Experimental}

Most of the potential measurements were conducted at Usujiri Fisheries Laboratory, Hokkaido University. A rectangular FRP tank with $2.3 \mathrm{~m}$ in length, $1.2 \mathrm{~m}$ in width, and $0.6 \mathrm{~m}$ in depth was used as an electrochemical cell for the potential measurement. $500 \mathrm{dm}^{3}$ of filtrated natural seawater (or seawaters diluted with deionized water) was poured into the tank. Two kinds of diluted seawaters were prepared: $1 / 5$ dilution and $1 / 10$ dilution. Filtrated natural seawater was pumped up from the coast near the laboratory though a cloth filter, and the salinity of the original seawater was $3.2 \%$. A zincked iron wire with $4 \mathrm{~mm}$ in diameter was used as a simulated structure to be cathodically protected. The wire is supplied from Shin-ei Kogyou, Co. Ltd., Japan, and the grade of the steel material for the wire is of JIS G3505 SWRM6 the chemical analytical data on which are presented in Table 1 . The commercially supplied wire was cut short to obtain three kinds of wires with the lengths of $L=1,1.5,2 \mathrm{~m}$. A cut wire was straightened, and dezincked by immersing in $0.1 \mathrm{~mol} / \mathrm{dm}^{3} \mathrm{HCl}$ solutions. A leading wire was soldered at one of the ends. After polished with an abrasive paper $(\mathrm{CC} 1200 \mathrm{CW}$ supplied from Nippon Coated Abrasive, Japan), the straightened wire was placed horizontally in the $500 \mathrm{dm}^{3}$ seawater along the longitudinal wall of the FRP tank. The seawater temperature was about $20^{\circ} \mathrm{C}$. At an appropriate position both horizontal and perpendicular to the middle point of the placed wire, a rod-type magnesium anode was fixed, vertically, which was the same kind as that used previously ${ }^{4)}$. A schematic arrangement of the electrodes in the tank was presented in Fig. 1. Using a galvanostat, a constant electric current was passed between the two electrodes, and the potentials of the anode $\left(E_{\mathrm{Mg}}\right)$ and the various points on the

Table 1 Composition of the steel material (JIS G3505 SWRM6) in the wire used for the cathode*).

\begin{tabular}{ccccc}
\hline $\mathrm{C}$ & $\mathrm{Si}$ & $\mathrm{Mn}$ & $\mathrm{P}$ & $\mathrm{S}$ \\
0.04 & 0.03 & 0.25 & 0.21 & 0.13 \\
\hline *) $\%$ balanced $\mathrm{Fe}$ & & &
\end{tabular}


wire cathode $\left(E_{\mathrm{i}}\right)$ were measured, using a saturated calomel electrode (SCE) as a reference electrode. The voltage $\left(V_{t}\right)$ applied by the galvanostat to maintain a constant electric current was also recorded. The potential measurements of the wire cathode were conducted at $20 \mathrm{~cm}$ intervals on the wire. The electric potential for any point either on anode or on cathode was very sensitive to the dynamic flow of seawater. This sensitivity increased with the salinity of seawater. In the case of the neat seawater with the salinity of $3.2 \%$, a stable potential value hardly could be obtained without any measure for the problem. Hence, a special care was taken to make the flow of seawater be as small as possible. The reference electrodes of SCE had been previously fixed at the respective points at which the potentials were to be measured. Because the readings of the potentials were unstable in the starting period of the experiment, the values at the electrodeposition time $=10$ min were simultaneously recorded for all the cases.

In the case of the cathode current density of $D_{\mathrm{k}}=1 \mathrm{~A} / \mathrm{m}^{2}$ and the cathode length of $L=2 \mathrm{~m}$, after finishing the potential measurement, the wire cathode was allowed to stand for 24 hours with keeping the constant current. Then, at every potential measuring point $\left(x=x_{\mathrm{i}}\right)$, the part of the formed calcareous deposit in both right and left $2.5 \mathrm{~cm}$ in respect of $x=x_{\mathrm{i}}$ on the wire cathode was dissolved with 1 $\mathrm{mol} / \mathrm{dm}^{3} \mathrm{HCl}$ solution. The amounts of magnesium and calcium in each of the solution were determined by a chemical analysis ${ }^{4)}$.

\section{Results and discussion}

\subsection{Examination of validity of Equation (5)}

4.1.1 Application of Equation (5) to the values of $V_{a}$

Fig. 2 shows the variation of the value of $V_{a}$ with the position of anode $\left(y_{0}\right)$ under a constant cathodic current

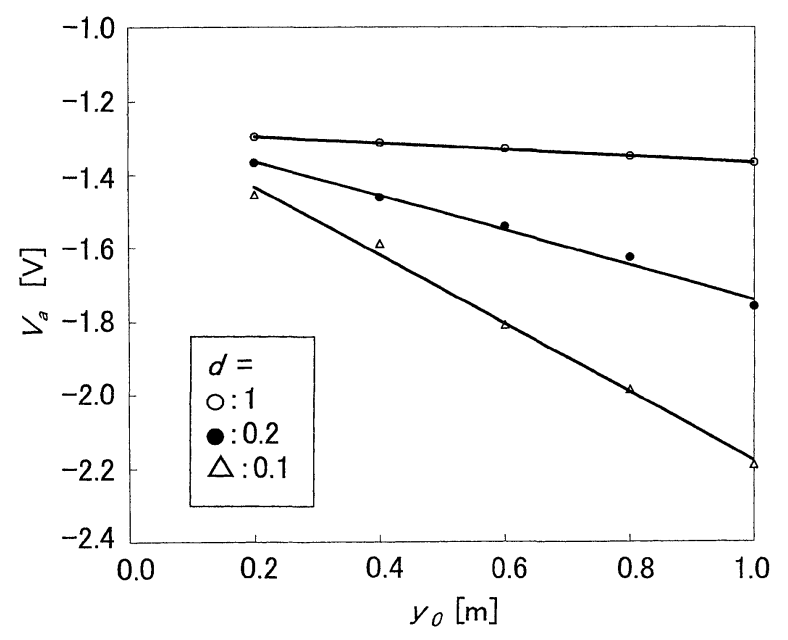

Fig. 2 The variation of $V_{\mathrm{a}}$ with the position of anode under a constant cathodic current density of $D_{\mathrm{K}}=0.5 \mathrm{~A} / \mathrm{m}^{2} . V_{\mathrm{a}}$ was measured for the seawater dilution ratios of $d=1(\mathrm{O}), 0.2$ $(\bullet)$ and $0.1(\triangle)$. density of $D_{\mathrm{K}}=0.5 \mathrm{~A} / \mathrm{m}^{2} . V_{\mathrm{a}}$ was measured for the seawater dilution ratios of $d=0.1,0.2$ and 1.0. For every case, the value of $V_{\mathrm{a}}$ decreases linearly with increasing $y_{0}$. Fig. 3 shows that the variation in the value of $E_{\mathrm{Mg}}$ plays only a small part in this variation in the value of $V_{\mathrm{a}}$ in Fig. 2 . Humble $^{2)}$ pointed out that solution potentials of $\mathrm{Mg}$ anodes are little affected by time or the rate of current out-put, because the corrosion products of $\mathrm{Mg}$ in seawater are nonadherent. This is partly the reason that this particular material has been mainly chosen as anode for the studies of this series. In accordance with the results by Humble ${ }^{2)}$, the anode potential, $E_{\mathrm{Mg}}$, remained almost constant when $d$ or $y_{0}$ was varied, as shown in Fig. 3 . Therefore, the variation of $V_{\mathrm{a}}$ with $d$ or $y_{0}$ observed in Fig. 2 is mainly responsible for the variation in the voltage, $V_{\mathrm{t}}$, impressed by the galvanostat to keep the electric current constant $\left(D_{\mathrm{K}}=0.5 \mathrm{~A} / \mathrm{m}^{2}\right)$.

The conversion of the real distance of $y_{0}$ in Fig. 2 to the hypothetical distance of $Y_{0}\left(=y_{0} / d\right)$ produces Fig. 4 . While three kinds of straight lines corresponding to three cases of the dilutions are necessary to represent all the data in Fig. 2, only a single line well does that in Fig. 4; all the data well fit to a single line (Fig. 4),

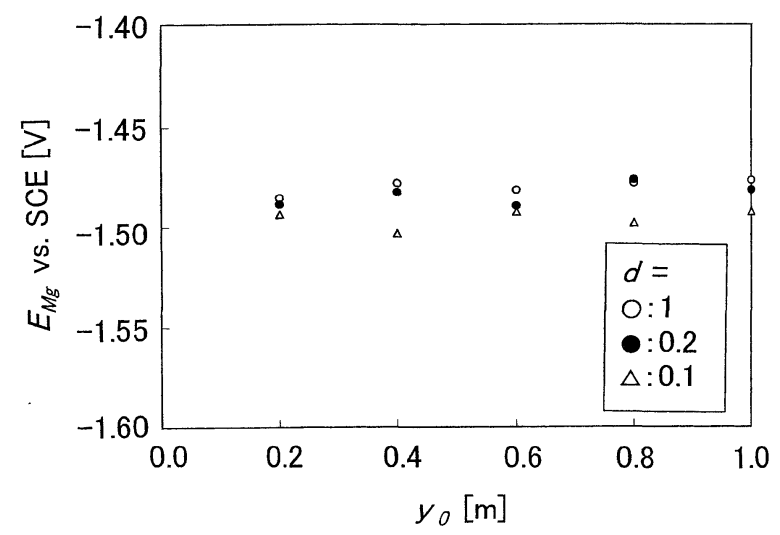

Fig. 3 The variation of $E_{\mathrm{Mg}}$ with the position of anode under a constant cathodic current density of $D_{\mathrm{K}}=0.5 \mathrm{~A} / \mathrm{m}^{2} . E_{\mathrm{Mg}}$ was measured for the seawater dilution ratios of $d=1$ $(\bigcirc), 0.2(\bigcirc)$ and $0.1(\triangle)$.

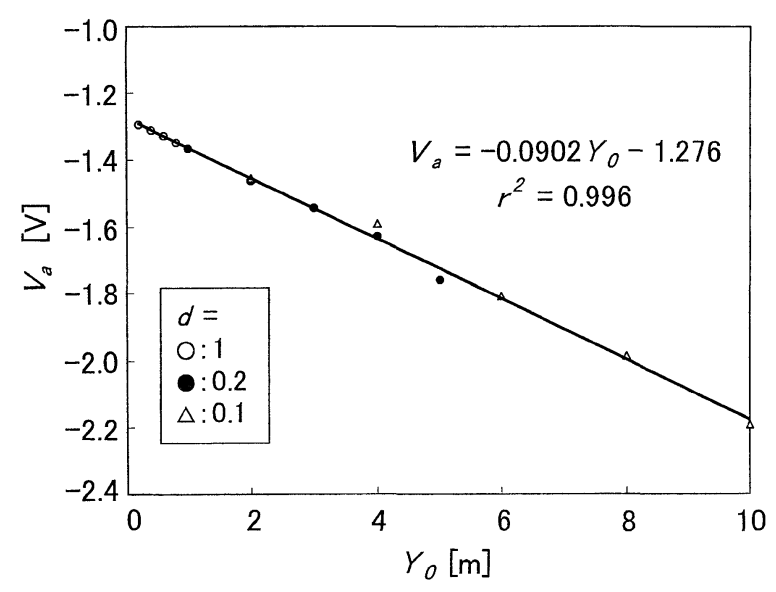

Fig. 4 The conversion of the real to the hypothetical distance for the data in Fig. 2. 


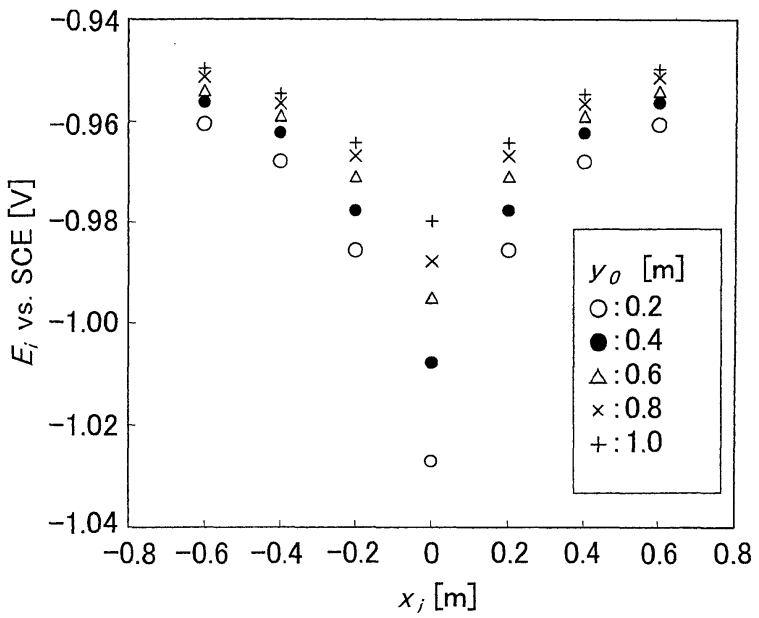

Fig. 5 The potential $\left(E_{\mathrm{i}}\right)$ distributions on the wire cathode for various anode positions $\left(y_{0}\right)$ at $d=1 . y_{0}=: 0,0.2 \mathrm{~m} ; 0,0.4$ $\mathrm{m} ; \triangle, 0.6 \mathrm{~m} ; \times, 0.8 \mathrm{~m} ;+, 1.0 \mathrm{~m}$.

$$
V_{\mathrm{a}}=-0.0902 Y_{0}-1.276
$$

and the value of $r^{2}$ is 0.996 , where $r$ is the correlation coefficient. This result supports the validity of the assumption made in deriving Equation (5), that is, the assumption that the electric resistance between the two electrodes in Fig. 1 chiefly comes from the part of seawater. In addition, this suggests that Equation (5) will become useful when we search proper anodes arrangement in the $\mathrm{CP}$ method for a gigantic marine structure.

\subsubsection{Application of Equation (5) to the values of $E_{\mathrm{i}}$}

Fig. 5 shows the potential distributions on the wire cathode (presented in Fig. 2) for various anode positions at $d$ $=1$. Marked unevenness in the distribution is observed around $x_{\mathrm{i}}=0$, and this unevenness becomes conspicuous as $y_{0}$ approaches 0 . The relation between $E_{\mathrm{i}}$ and $y_{0}$ at $x_{\mathrm{i}}=0$ in Fig. 5 can be represented by the semi-logarithmic equation,

$$
E_{\mathrm{i}}=0.0291 \ln y_{0}-0.980
$$

Semi-logarithmic plots of all the data on $E_{\mathrm{i}}$ for the dilution ratios of $d=1,0.2$ and 0.1 at $x_{\mathrm{i}}=0$ give three straight lines, as presented in Fig. 6 . At a given value of $y_{0}, E_{\mathrm{i}}$ increases with decreasing $d$. The three straight lines in Fig. 6 have almost the same slope, being different only in the intersection points with the vertical axis. The conversion of the real to the hypothetical distance for the data in Fig. 6 gives Fig. 7. Just as the case for $V_{a}$, the conversion puts together the three lines in Fig. 6 into the single one,

$$
E_{\mathrm{i}}=0.0254 \ln Y_{0}-0.982
$$

This equation is almost the same as Equation (7), and the fitting of all the data to this equation is excellent $\left(r^{2}=\right.$ 0.996). Until now, as an example, the dependence of $E_{\mathrm{i}}$ on $y_{0}$ has been examined, focusing on only a single position on the wire cathode, i.e., $x_{\mathrm{i}}=0$. This reason is that around this point the unevenness in the distribution of $E_{\mathrm{i}}$ becomes most marked. However, exactly similar discussion can be made irrespective of the value of $x_{\mathrm{i}}$, only if we neglect the difference of the dependency of $E_{\mathrm{i}}$ on $y_{0}$. As can readily be seen from Fig. 5, the farther a point on the wire cathode

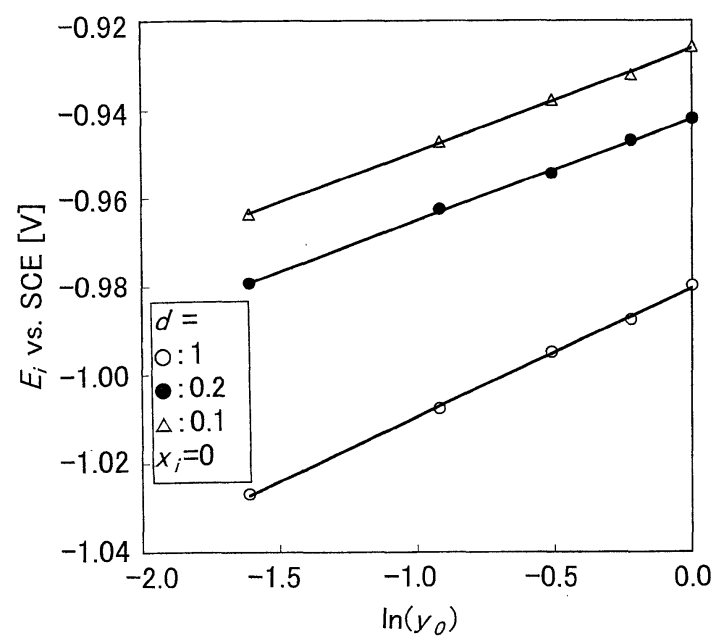

Fig. 6 Semi-logarithmic plots of $E_{\mathrm{i}}$ for all the dilution ratios of $d=$ $1(\bigcirc), 0.2(\bigcirc)$ and $0.1(\triangle)$ at $x_{\mathrm{i}}=0$.

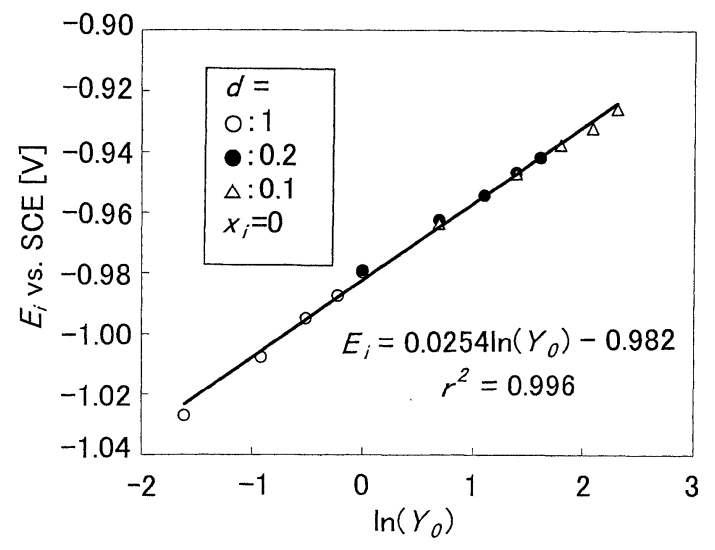

Fig. 7 The conversion of the real to the hypothetical distance for the data in Fig. 6.

was deviate from the midpoint, the weaker became the dependency of $E_{\mathrm{i}}$ on $y_{0}$.

All the facts above described show the validity of utilizing Equation (5) as a method of searching proper arrangement of anodes, when we try to protect a gigantic marine structure cathodically.

\subsection{Method of utilizing Equation (5) to search a proper anodes arrangement}

Until now, one more problem has been left unsolved; utilizing the scale-up Equation (5), how to obtain the necessary number of anodes and their proper arrangement for the cathodic protection of a gigantic marine structure. Below, we will consider this left problem.

\subsubsection{Values of cathode potentials}

To solve this problem, the data on cathode and anode potentials were collected at $d=0.1$ for various $L, D_{\mathrm{k}}, y_{0}$ and $x_{\mathrm{i}}$ values. The examined factors are summarized in Table 2 . The number of all the combinations of the factors is over 3000 , but some combinations were left out.

The potential distributions on wire cathodes are summarized in Figs. 8, 9 and 10, which correspond to the cases of 
Table 2 Experimental variables.

\begin{tabular}{c|l}
\hline Factor & \multicolumn{1}{|c}{ Experiment condition } \\
\hline$L[\mathrm{~m}]$ & $1,1.5,2$ \\
$D_{\mathrm{k}}\left[\mathrm{A} / \mathrm{m}^{2}\right]$ & $0.2,0.5,1.0,1.5,2$ \\
$Y_{0}[\mathrm{~m}]$ & $0.05,0.1,0.15,0.2,0.3,0.4,0.6$ \\
$X_{\mathrm{i}}[\mathrm{m}]$ & $0.2 \mathrm{~m}$ interval \\
\hline
\end{tabular}

$D_{\mathrm{k}}=0.5,1.0$ and $2.0 \mathrm{~A} / \mathrm{m}^{2}$, respectively. From these results, it seems that the unevenness of cathode potential distribution increases with decreasing $L$, decreasing $Y_{0}$, or increasing $D_{\mathrm{k}}$, provided that the other factors are kept constant. In particular, this unevenness becomes marked around $X_{\mathrm{i}}=0$ as indicated by dotted lines in the figures. However, for a quantitative discussion of this kind, a rigorous definition is necessary of the term "unevenness" itself. This will be discussed in detail, later.

\subsubsection{Values of $V_{a}$}

The results of $V_{\mathrm{a}}$ measurements have been collected in Fig. 11. The results can be summarized as follows.

- The value of $V_{\mathrm{a}}$ decreases linearly with increasing $Y_{0}$.

- The longer the wire cathode length, $L$, is, the larger the above decreasing rate of $V_{\mathrm{a}}$ becomes.

- The same as above stated can also be said about $D_{\mathrm{k}}$ instead of $L$.

\subsubsection{Calcareous deposit distribution on the cath- ode}

The results of calcareous deposition on the wire cathode
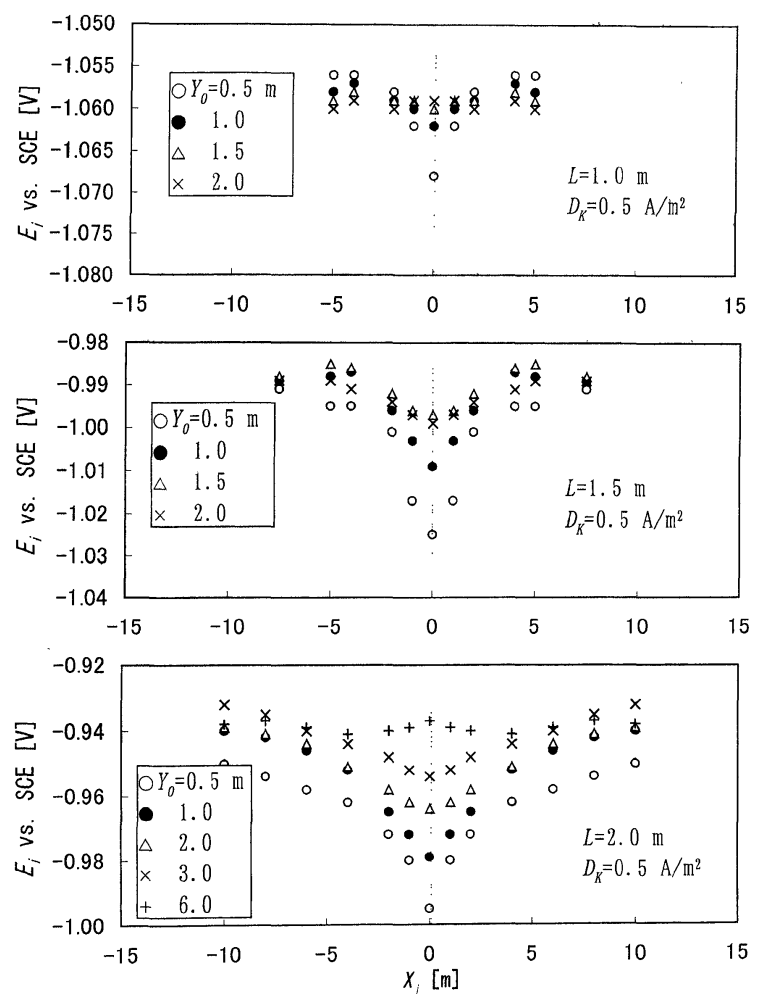

Fig. 8 Potential distribution of cathode for $D_{\mathrm{k}}=0.5 \mathrm{~A} / \mathrm{m}^{2} . E_{\mathrm{i}}$ denotes the potential at the point of $X_{\mathrm{i}}\left(=x_{\mathrm{i}} / d\right)$ on the wire cathode. Each symbol represents the hypothetical position of the anode, $Y_{0} \mathrm{~m}$.
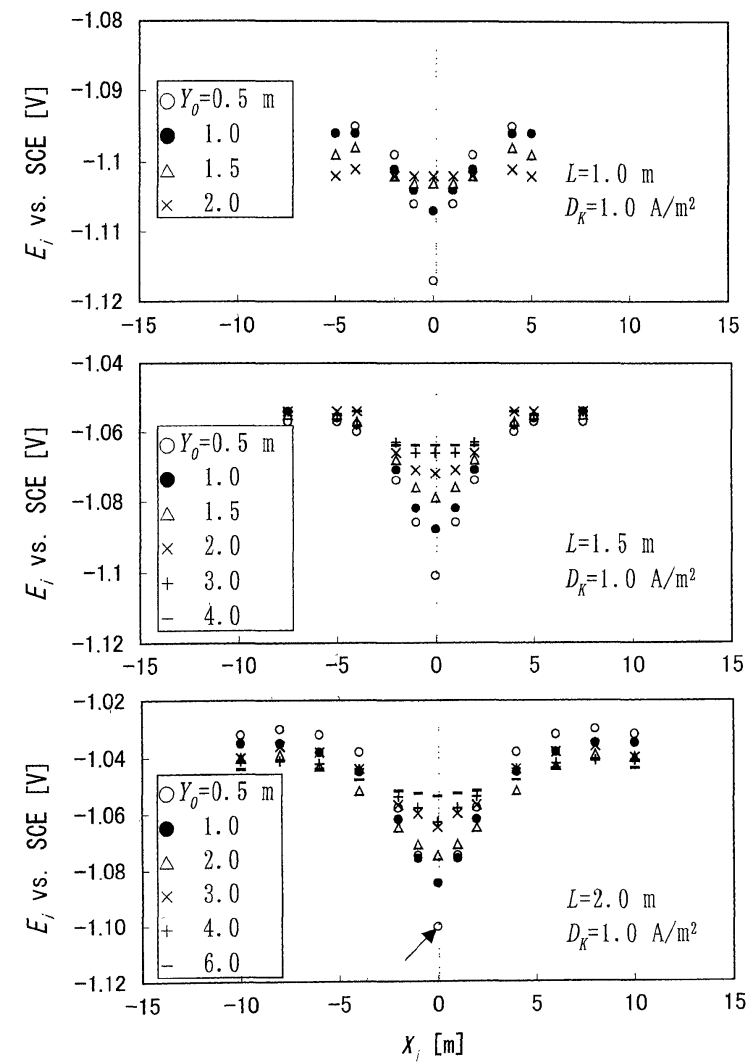

Fig. 9 Potential distribution of cathode for $D_{\mathrm{k}}=1 \mathrm{~A} / \mathrm{m}^{2} . E$ denotes the potential at the point of $X_{\mathrm{i}}\left(=x_{\mathrm{i}} / d\right)$ on the wire cathode. Each symbol represents the hypothetical position of the anode, $Y_{0} \mathrm{~m}$.
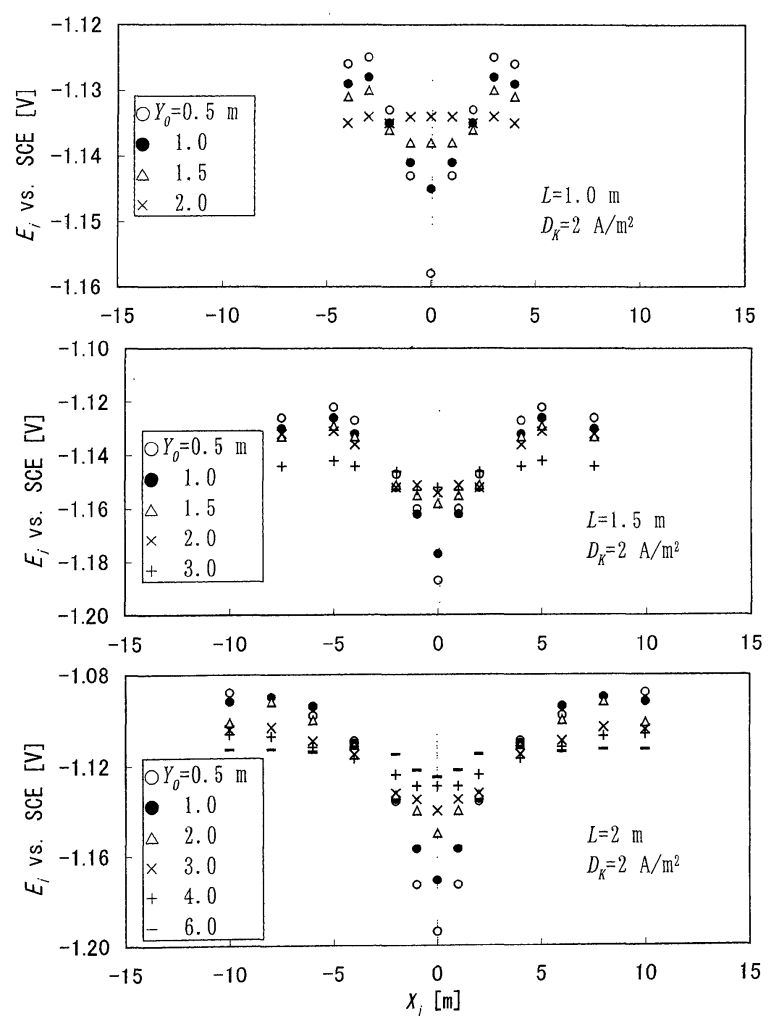

Fig. 10 Potential distribution of cathode for $D_{\mathrm{k}}=2 \mathrm{~A} / \mathrm{m}^{2} \cdot E_{\mathrm{i}}$ denotes the potential at the point of $X_{\mathrm{i}}\left(=x_{\mathrm{i}} / d\right)$ on the wire cathode. Each symbol represents the hypothetical position of the anode, $Y_{0} \mathrm{~m}$. 

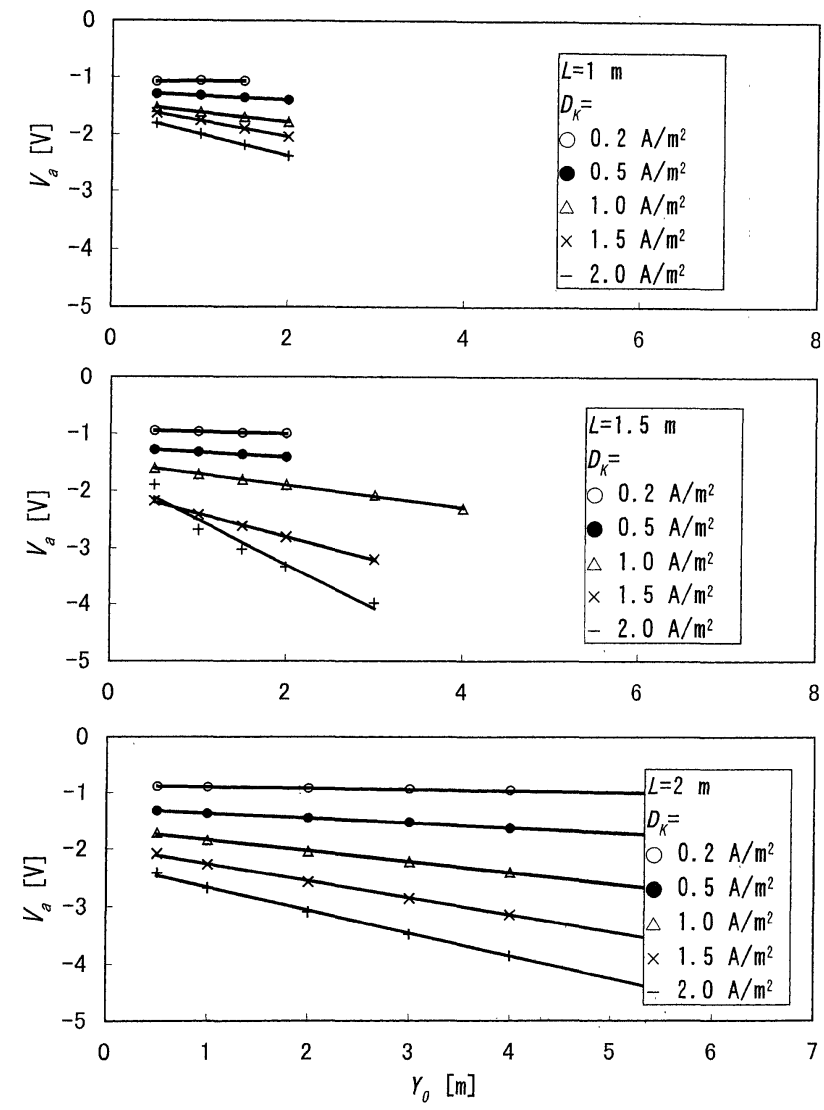

Fig. 11 Dependence of $V_{\mathrm{a}}$ on the anode position, $Y_{0}\left(=y_{0} / d\right)$. The keys for symbols are presented in the figure.

are presented in Fig. 12. The results were obtained from the cathodic protection experiments carried out under the conditions of $D_{\mathrm{k}}=1 \mathrm{~A} / \mathrm{m}^{2}, L=2 \mathrm{~m}$, the time of electrodeposition, $t=24 \mathrm{~h}$, and the various values of $Y_{0}$. The unevenness of the deposit distribution throughout the structure seems to become marked with decreasing $Y_{0}$. This unevenness is particularly pronounced around $X_{\mathrm{i}}=0$ as indicated by a dotted line in the figure.

\subsubsection{Relation between deposit distribution and current density distribution}

Since cathodic current is the direct cause for the production of calcareous deposit on a cathode through the electrochemical reactions,

$$
\begin{aligned}
& \mathrm{H}_{2} \mathrm{O}+\frac{1}{2} \mathrm{O}_{2}+2 \mathrm{e} \rightarrow 2 \mathrm{OH}^{-} \\
& 2 \mathrm{H}_{2} \mathrm{O}+2 \mathrm{e} \rightarrow 2 \mathrm{OH}^{-}+\mathrm{H}_{2} \\
& \mathrm{HCO}_{3}{ }^{-}+\mathrm{OH}^{-} \rightarrow \mathrm{CO}_{3}{ }^{2-}+\mathrm{H}_{2} \mathrm{O} \\
& \mathrm{CO}_{3}{ }^{2-}+\mathrm{Ca}^{2+} \rightarrow \mathrm{CaCO}_{3} \\
& \mathrm{Mg}^{2+}+2 \mathrm{OH}^{-} \rightarrow \mathrm{Mg}(\mathrm{OH})_{2}
\end{aligned}
$$

we should discuss the problem of this distribution in terms of the distribution of the cathodic current density on the cathode, but not that of the potential. From Equation (5), the potential distributions in Figs. 8, 9 and 10 can readily be converted to the corresponding cathodic current density distributions. And using these distributions, we can readily obtain the relation between the experimental deposit amount $\left(W_{\mathrm{T}}\right)$ and

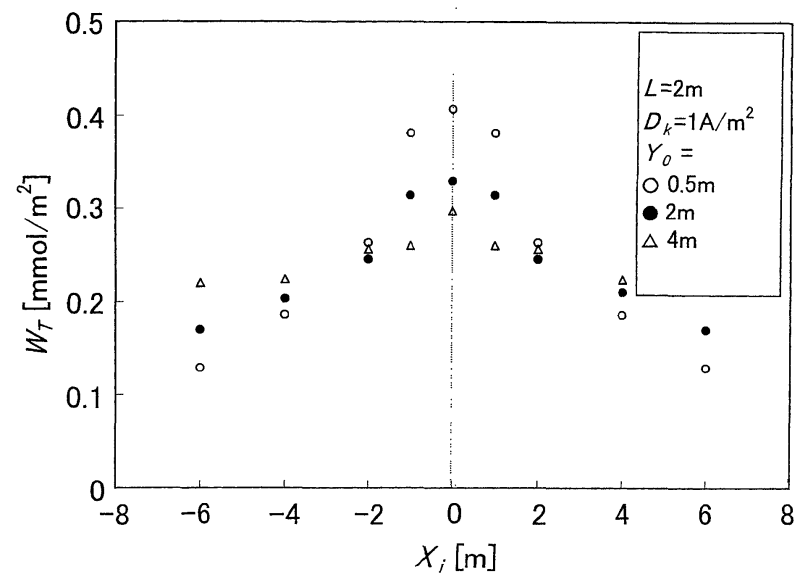

Fig. 12 Distribution of calcareous deposit amount $\left(W_{\mathrm{T}}\right)$ on the wire cathode with the length $L=2 \mathrm{~m}$ at $D_{\mathrm{k}}=1 \mathrm{~A} / \mathrm{m}^{2}$. The keys for the symbols are found in the figure.

the calculated one $\left(W_{\text {cld }}\right)$, as presented in Fig. 13, where assumption was made that the current efficiency be $100 \%$ by

$$
W_{\text {cld }}=\frac{i_{\mathrm{i}}(\pi D \Delta L) t}{2 F}
$$

where $t$ and $F$ are the electrodeposition time and the Faraday constant, respectively. $D$ is the diameter of the wire cathode (in the present case, $D=4 \mathrm{~mm}$ ), and $\Delta L$ the partial length of the wire the deposit on which was dissolved (in the present case, $\Delta L=5 \mathrm{~cm}$ ). From Fig. 13, it can be seen that there is a close relation between $W_{\mathrm{T}}$ and $W_{\text {cld }}$, and but for the datum indicated by an arrow, the relation can approximately be represented by a straight line passing through the origin. The datum that greatly deviates from the line corresponds to the deposit formed on the cathode at $X_{\mathrm{i}}=0, Y_{0}=0.5 \mathrm{~m}, D_{\mathrm{K}}=1.0 \mathrm{~A} / \mathrm{m}^{2}$ and $L=2 \mathrm{~m}$. Under these conditions, the most pronounced unevenness was produced in the cathode potential distribution for the cath-

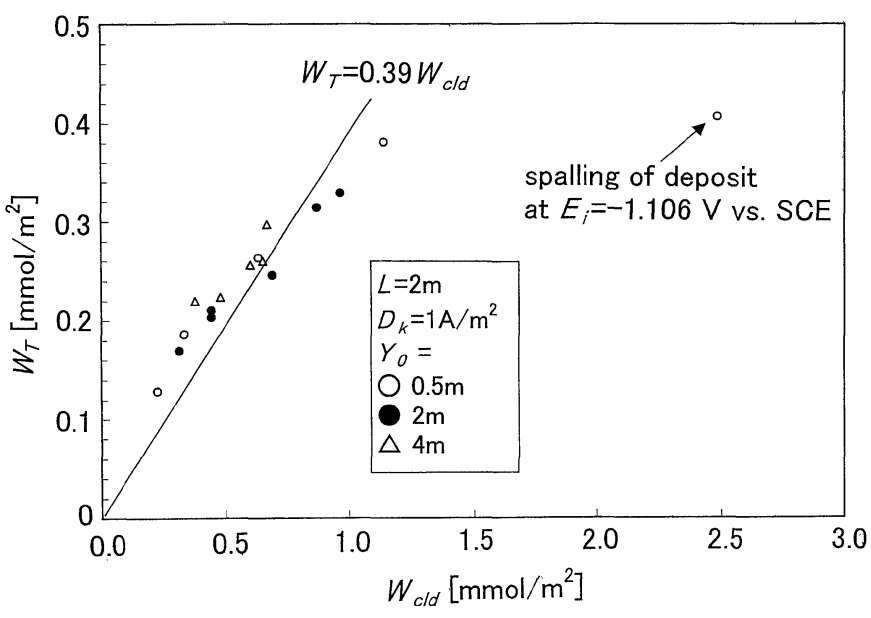

Fig. 13 The relation between the experimental deposit amount $\left(W_{\mathrm{T}}\right)$ and the deposit amount calculated from the current density on the assumption that the current efficiency be $100 \%\left(W_{\text {cld }}\right)$. The explanation for the data indicated by the arrow is found in the text. 
ode of $L=2 \mathrm{~m}$ at $D_{\mathrm{K}}=1.0 \mathrm{~A} / \mathrm{m}^{2}$ (the datum indicated by an arrow in the bottom part of Fig. 9), and as negative as the potential, $E_{\mathrm{i}}=-1.106 \mathrm{~V}$ vs. SCE, was attained. It has been reported $^{4)}$ that under $E_{\mathrm{i}}=-1.1 \mathrm{~V}$ vs. SCE or more negative, the cathodic electrochemical reaction (10) begins to operate, and the significant spalling of calcareous deposits due to the hydrogen evolution occurs. In fact, the spalling of deposits under these conditions could readily be confirmed by a visual inspection. Therefore, if we made an adequate allowance for the loss in deposit amount due to this spalling, all the data would better obey a linear relation. In Fig. 13, the slope of the straight line gives the current efficiency for the electrodeposition as $39 \%$. This value is considerably small as compared with the value in the previous paper $(60 \%)^{1)}$.

\subsubsection{Quantitative criterion of unevenness in cal- careous deposit distribution and procedure function for anodes arrangement}

The presence of a linear relationship between $W_{\mathrm{T}}$ and $W_{\text {cld }}$ shows that the distribution of $W_{\mathrm{T}}$ on a cathode can be attributed to that of $i_{\mathrm{i}}$. An advantage of this attribution is in the point that it excludes the possibility of some disturbance in the distribution due to the spalling of deposit. Therefore, hereafter, we will discuss the unevenness of calcareous deposit distribution in terms of $i_{\mathrm{i}}$. Let the quantitative unevenness of the current density distribution be defined by

$$
U=\frac{\sqrt{\sum\left(i_{\mathrm{i}}-\bar{i}\right)^{2} / N}}{\bar{i}}
$$

where $N$ is the number of the data, and $\bar{i}$ is the mean value of $i_{\text {, }}$, i.e.,

$$
\bar{i}=\frac{\pi D \int_{-x_{e}}^{x_{\mathrm{e}}} i_{\mathrm{i}} d x}{\pi D L}=D_{\mathrm{k}}
$$

The denominator should simply be equal to the total electric current passed through the cathode, and, of course, a fixed value for a particular electrodeposition experiment under a galvanostatic mode. This was confirmed by the numerical integration in the denominator of Equation (16) for all the current density distributions in this paper.

$U$ is a dimensionless number, and gives a quantitative criterion upon which to judge whether or not certain unevenness in the distribution of a calcareous deposit formed under a particular arrangement of electrodes is tolerable. The 44 potential distribution curves in Figs. 8, 9 and 10 can be converted to the 44 current density distributions by the use of Equation (5). Further, from these distributions, we can calculate the 44 values of $U$, using Equation (15).

Next, the unevenness of the electrodes arrangement is defined as the degree of the flatness in the isosceles triangle formed with $-x_{\mathrm{e}}, x_{\mathrm{e}}$ and $y_{0}$ in Fig. 1 , that is, $L / y_{0}=$ $L / d Y_{0}$. The term, $L / d$ simply corresponds to the hypothetical length of a structure. Plotting the previously obtained 44 values of $U$ against $L / d Y_{0}$ gives Fig. 14. Although these

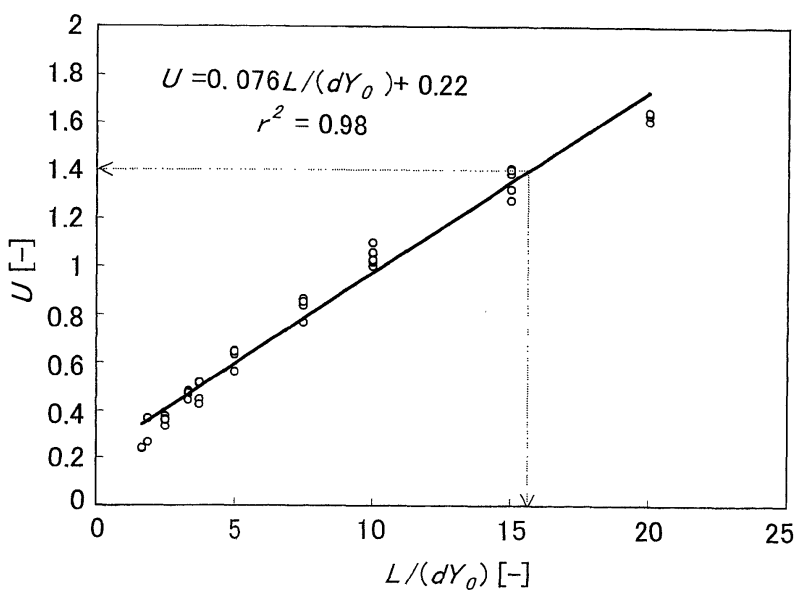

Fig. 14 The relation between the dimensionless number for the unevenness in calcareous deposit distribution, $U$, and the dimensionless number for the structure length, $L / d Y_{0}$.

44 values of $U$ come from the electrodeposition experiments under various conditions of $D_{\mathrm{k}}, L$ and $Y_{0}$, they can all be well represented by only the single linear equation,

$$
U=0.076\left(L / d Y_{0}\right)+0.22
$$

This is a kind of procedure function for designing proper electrodes arrangement in the $\mathrm{CP}$ of gigantic marine structure accompanied with calcareous deposits. Using this equation, we can readily estimate various design parameters for the cathodic protection of a marine structure. For example, we may suppose to have a structure $(L=300 \mathrm{~m})$ in natural seawater $(d=1)$ to be cathodically protected with a calcareous deposit the tolerable unevenness of which must be less than $U=1.4$. Because this value of $U$ corresponds to $L / d Y_{0}=15.06$ (see Fig. 14), assuming $y_{0}=1$ $\mathrm{m}$, it is estimated that more than $300 / 15.06 \doteqdot 20$ anodes must be arranged on a line $1 \mathrm{~m}$ apart from and parallel to the structure at regular intervals.

Various simplifications were made in deriving Equation (17). First, the data were obtained from experiments under quiescent seawater. It is well known that the dynamic flows of seawater give significant effects on cathodic polarization behavior and the properties of calcareous deposits. Second, unlike the straight structure used in the present article, the geometrical complexity of a real structure adds to the difficulty of actual problems. Finally, the data on cathode potential were collected in the initial stage of electrodeposition. Prolongation of electrodeposition time makes the amount of calcareous deposit formed on the cathode increase, and this in turn may influence the potential distribution. In spite of these simplifications, Equation (17) will play an important and fundamental part in the design of cathodically protecting a gigantic marine structure.

\section{Conclusions}

To estimate a proper anodes arrangement in the cathodic protection of a gigantic marine structure, quite a simple 
method (dilution method) was proposed, and the validity and applicability of the method was examined. The results can be summarized as follows.

1. As far as potential distributions along the wire cathodes studied in this paper are concerned, the dilution of seawater with fresh water corresponded to the geometrical extension of the system under consideration, that is, Equation (5) held well.

2. At a fixed position of the anode, there arose marked unevenness around the midpoint in the cathode potential distribution along the wire cathode.

3. The plotting of the unevenness in the electric current density distribution defined by Equation (15) against the unevenness of the electrodes arrangement, $L / d Y_{0}$ $\left(=L / y_{0}\right)$, gave a linear relation (Equation (17)).

4. The equation (Equation (17)) is a kind of procedure function for designing proper electrodes arrangement in the $\mathrm{CP}$ of gigantic marine structures accompanied with calcareous deposition, and can predict various design parameters concerning the proper electrodes arrangement.

\section{Acknowledgment}

This work was partially supported by a Grant-in-Aid for Scientific Research No.15360457 from the ministry of Education, Culture, Sports, Science and Technology, Japan.

\section{References}

1) K. Akamine and I. Kashiki, Zairyo-to-Kankyo, 53, 354 (2004).

2) R. A. Humble, Corrosion, 4, 358 (1948).

3) G. Salvago and G. Bollini, Corrosion, 55, 397 (1999).

4) K. Akamine and I. Kashiki, Zairyo-to-Kankyo, 51, 496 (2002).

5) D. R. Crow, "Principles and Application of Electrochemistry, 3rd Ed.”, London, Blackie Academic \& Professional, Chap. 7 (1988)

6) W. Wang, W. H. Hartt and S. Chen, Corrosion, 52, 419 (1996).

7) Denki-kagaku-kyoukai (ed.), "Denki-kagaku-benran (Handbook of Electrochemistry), 4th Ed.", Maruzen, Tokyo, p.263 (1993) (in Japanese).

8) F. G. W. Smith (Ed.), "Handbook of Marine Science, Vol.1", Ohio, CRC Press, p.67 (1974).

(Manuscript received September 11, 2003; in final form March 22, 2004) 